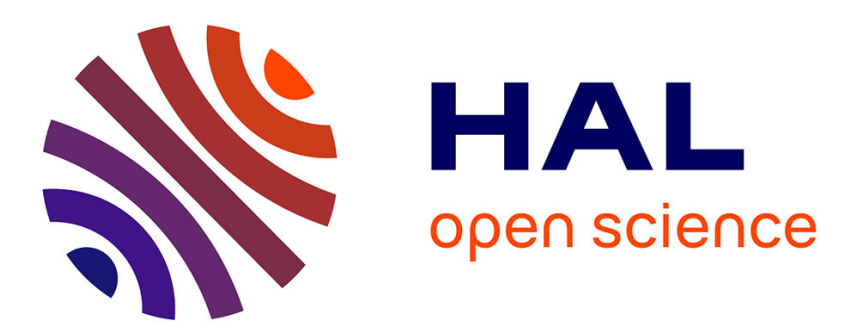

\title{
Enhanced photosensitivity of electro-oxidized epitaxial graphene
}

Mikhail E. Itkis, Feihu Wang, Palanisamy Ramesh, Elena Bekyarova, Sandip Niyogi, Xiaoliu Chi, Claire Berger, Walt A. de Heer, Robert C. Haddon

\section{- To cite this version:}

Mikhail E. Itkis, Feihu Wang, Palanisamy Ramesh, Elena Bekyarova, Sandip Niyogi, et al.. Enhanced photosensitivity of electro-oxidized epitaxial graphene. Applied Physics Letters, 2011, 98 (9), pp.093115. 10.1063/1.3562316 . hal-01002904

\section{HAL Id: hal-01002904 https://hal.science/hal-01002904}

Submitted on 7 Jun 2014

HAL is a multi-disciplinary open access archive for the deposit and dissemination of scientific research documents, whether they are published or not. The documents may come from teaching and research institutions in France or abroad, or from public or private research centers.
L'archive ouverte pluridisciplinaire HAL, est destinée au dépôt et à la diffusion de documents scientifiques de niveau recherche, publiés ou non, émanant des établissements d'enseignement et de recherche français ou étrangers, des laboratoires publics ou privés. 


\title{
Enhanced photosensitivity of electro-oxidized epitaxial graphene
}

\author{
Mikhail E. Itkis, ${ }^{1}$ Feihu Wang ${ }^{1}$ Palanisamy Ramesh, ${ }^{1}$ Elena Bekyarova, ${ }^{1}$ Sandip Niyogi, ${ }^{1}$ \\ Xiaoliu Chi, ${ }^{1, a}$ Claire Berger, ${ }^{2,3}$ Walt A. de Heer, ${ }^{2}$ and Robert C. Haddon ${ }^{1, b)}$ \\ ${ }^{1}$ Departments of Chemistry, Chemical and Environmental Engineering and Physics, Center for Nanoscale \\ Science and Engineering, University of California, Riverside, California 92521, USA \\ ${ }^{2}$ School of Physics, Georgia Institute of Technology, Atlanta, Georgia 30332, USA \\ ${ }^{3}$ CNRS-Institute Néel, BP 166, 38042 Grenoble, Cedex 9, France
}

(Received 21 November 2010; accepted 14 February 2011; published online 3 March 2011)

\begin{abstract}
We report the enhanced photosensitivity of epitaxial graphene (EG) after electrochemical oxidation in nitric acid. The onset of photoconductivity appears at a photon energy of $\sim 1.7 \mathrm{eV}$ while the responsivity reaches $2.5 \mathrm{~A} / \mathrm{W}$ at a wavelength of $470 \mathrm{~nm}$ (blue light, energy $2.64 \mathrm{eV}$ ) and further increases to $200 \mathrm{~A} / \mathrm{W}$ in the UV spectral range $(3.5 \mathrm{eV}, 350 \mathrm{~nm})$. The observed photoresponse is attributed to the formation of deep traps at the electro-oxidized EG interface, which release charge carriers under illumination and prolong the life time of the photocarriers. Potential applications of electro-oxidized EG in ultraviolet photodetection are discussed. (C) 2011 American Institute of Physics. [doi:10.1063/1.3562316]
\end{abstract}

Graphene is a fascinating material with unique electronic properties. ${ }^{1-3}$ Pristine graphene has no energy gap; nevertheless the photoelectronic properties of graphene have attracted significant interest because of the exciting physics and potential for photonic applications. ${ }^{4,5}$ Despite the extremely short life time of the photocarriers, photoconductivity in graphene was detected using a laser beam focused at regions adjacent to the metal electrodes, where the internal electric field separates the photogenerated electron-hole pairs before they recombine. ${ }^{4-7}$ As a result, an ultrafast graphene photodetector has been demonstrated with an operating bandwidth of $40 \mathrm{GHz}$, and a responsivity of $6.1 \mathrm{~mA} / \mathrm{W}$ at a wavelength $\lambda$ of $1.55 \mu \mathrm{m} .{ }^{4,5}$ Photoconductivity was also observed in thin films of reduced graphene oxide ${ }^{8,9}$ and enhanced by attachment of GdSe quantum dots, resulting in extrinsic photoconductivity with responsivity up to $4 \mathrm{~mA} / \mathrm{W}(\lambda$ $=532 \mathrm{~nm}){ }^{10,11}$

Band gap chemical engineering has been utilized to modify the electronic and optical properties of single-walled carbon nanotubes (SWNTs), ${ }^{12}$ and chemistry is expected to be effective in controlling the electronic structure of graphene by inducing an energy gap. ${ }^{13,14}$ In this letter, we report that electrochemical oxidation increases the photoresponsivity of epitaxial graphene (EG) to the level 2.5 $\mathrm{A} / \mathrm{W}$ under blue light $(\lambda=470 \mathrm{~nm})$ and $>200 \mathrm{~A} / \mathrm{W}$ in the UV range, which exceeds previously reported responsivity values $^{6-8,10,11}$ by several orders of magnitude.

For this work we employed multilayer EG grown on the C-phase of a $4 \mathrm{H}-\mathrm{SiC}$ wafer (Cree Inc.) by hydrogen etching and vacuum graphitization at $\sim 1450{ }^{\circ} \mathrm{C}$ at $1 \times 10^{-10}$ Torr. ${ }^{1,3}$ Raman spectra of pristine EG show prominent G- and 2Dbands at frequencies of $1580 \mathrm{~cm}^{-1}$ and $2694 \mathrm{~cm}^{-1}$, respectively, confirming the presence of graphene layers on the $\mathrm{SiC}$ surface. Two identical dog bone shaped EG test and reference channels, with length $1.2 \mathrm{~mm}$ and width $0.35 \mathrm{~mm}$ were patterned side-by-side on the same wafer $(3.5 \times 4.5 \mathrm{~mm})$.

\footnotetext{
${ }^{a}$ Permanent address: Department of Chemistry, Texas A\&M University, Kingsville, Texas 78363, USA.

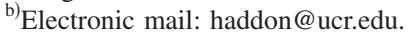

The electrical contacts to the channels were formed by e-beam evaporation of $10 \mathrm{~nm} \mathrm{Pd}$ and $100 \mathrm{~nm}$ Au layers. Electrochemical oxidation utilizing $1 \mathrm{M}$ nitric acid electrolyte was applied to the test channel [Fig. 1(a)], following a previously described procedure. ${ }^{15}$ The electro-oxidation was conducted in potentiostatic mode by applying $+0.8 \mathrm{~V}$ to the test channel (working electrode) against a Pt wire electrode inserted into the electrolyte drop. The size of the drop $\sim 1.5 \mathrm{~mm}$ was controlled by a capillary Teflon tube. [Fig. 1(a)]. Raman spectra showed that with increasing the oxidation time, the intensities of $\mathrm{G}$ and $2 \mathrm{D}$ bands decreased and the $\mathrm{D}$ band at $1340 \mathrm{~cm}^{-1}$ was enhanced [Fig. 1(b)]. After electro-oxidative removal of three to four EG layers out of the initial six to eight graphene layers, the resistance of the EG channel increased by a factor of 2 .

Initially, enhanced photoconductivity was observed when the EG sample was exposed to the fluorescent room light: under the light the resistance of the electro-oxidized (EO) channel decreases by $\sim 6 \%$ while the changes in resis-

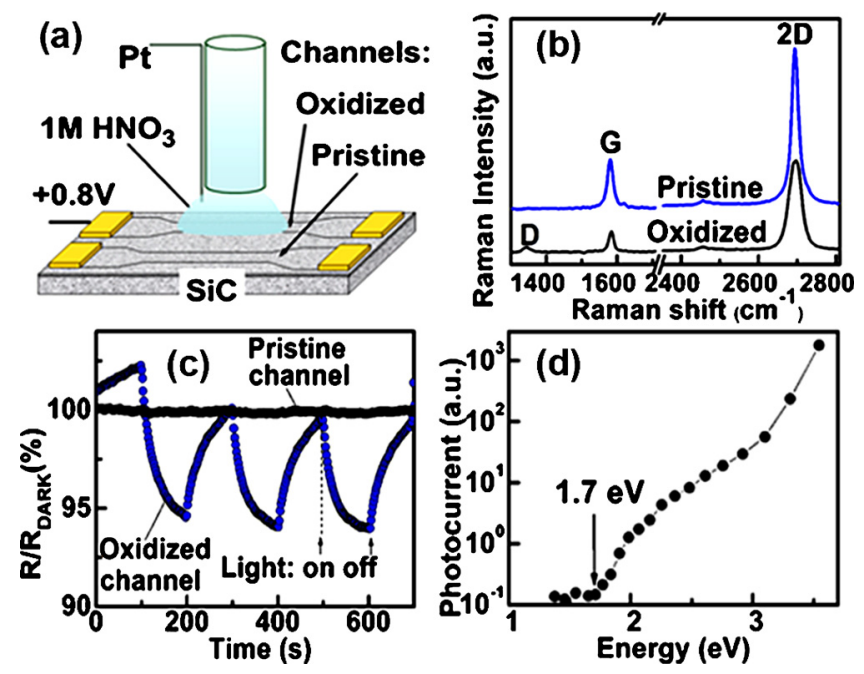

FIG. 1. (Color online) (a) Schematic of electro-oxidation experiment; (b) Raman spectra of pristine and EO-EG $\left(\lambda_{\text {laser }}=532 \mathrm{~nm}\right)$; (c) effect of fluorescent room light illumination on the resistance of EO and pristine EG channels. (d) Spectral dependence of photoresponse of the EO channel. 

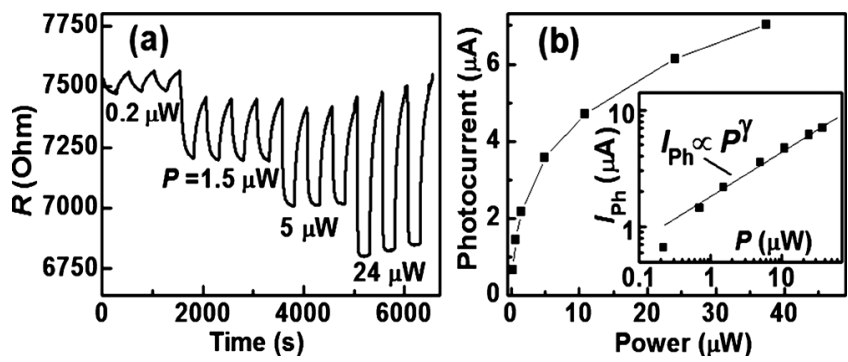

FIG. 2. (a) Modulation of the resistance of the EO-EG channel under square-wave pulses of blue LED illumination of different power. (b) Dependence of EO-EG channel photocurrent $I_{\mathrm{Ph}}$ on the power $P$ of incident radiation. Inset shows the same dependence in double-logarithmic scales.

tance of the reference (pristine) channel were two orders of magnitude lower [Fig. 1(c)]. The spectral dependence of the observed photoconductivity was measured utilizing a stepby-step scanning technique in which the spectrophotometer resides at each wavelength for a sufficient time to allow several on-off light cycles to accommodate the slow transient character of the photoresponse. The spectral photoresponse was normalized to the intensity of the source utilizing a spectrally flat pyroelectric detector. Figure 1(d) presents the spectral dependence of the photoconductivity showing the onset at a photon energy $\sim 1.7 \mathrm{eV}$ followed by an increase in the photoresponse by four orders of magnitude in the UV range.

Figure 2(a) shows the photoresponse of the EO-EG channel to square-wave pulses of blue light-emitting diode (LED) irradiation $(\lambda=470 \mathrm{~nm})$ : the relative change in resistance under the incident power of $0.2 \mu \mathrm{W}$ is at the level of $1 \%$ with a signal to noise ratio of $\sim 100$. At incident power of $0.2 \mu \mathrm{W}$ and channel bias of $450 \mathrm{mV}$, we obtained a current responsivity $\sim 2.5 \mathrm{~A} / \mathrm{W}$ and voltage responsivity $\sim 20000 \mathrm{~V} / \mathrm{W}$ (measured against a calibrated Si photodiode), which is three to four orders of magnitude higher than previously reported values in graphene. ${ }^{4,5,7}$ The current responsivity increases by more than two orders of magnitude in the UV range $(\lambda=350 \mathrm{~nm})$, where it exceeds $200 \mathrm{~A} / \mathrm{W}$ [Fig. 1(d)]. The contribution of the $4 \mathrm{C}-\mathrm{SiC}$ substrate (band gap $3.2 \mathrm{eV}$ ) to the photoconductivity in the UV range can be excluded because the photoresponse of the pristine channel (also supported on a SiC substrate), constitutes less than $1 \%$ of the photoresponse of the oxidized channel. The characteristic photoresponse time is in the tens of seconds; it persists for extended periods of time after the irradiation is turned off; thus, it can be classified as transient photoconductivity. ${ }^{16}$

On the basis of the weak temperature dependence of resistance of the EO-EG channel, analyzed in detail in our previous publication, ${ }^{15}$ we concluded that the electrooxidation does not induce an energy gap at the Fermi level. ${ }^{15}$ Thus, the enhanced photoresponse in EO-EG cannot be related to intrinsic photoconductivity due to interband excitation of the electron-hole pairs; rather we attribute this effect to formation of localized defect states at the EG/EO-EG interface from which photogenerated carriers are released and enter the EG channel thus giving rise to the observed photoconductivity. The interface states also change the recombination dynamics by preferentially capturing one carrier type before the electron-hole pairs recombine, temporarily localizing these carriers in the traps and later re-injecting them into the channel, thereby extending the carriers life time from the (intrinsic) picosecond time scale to some tens of
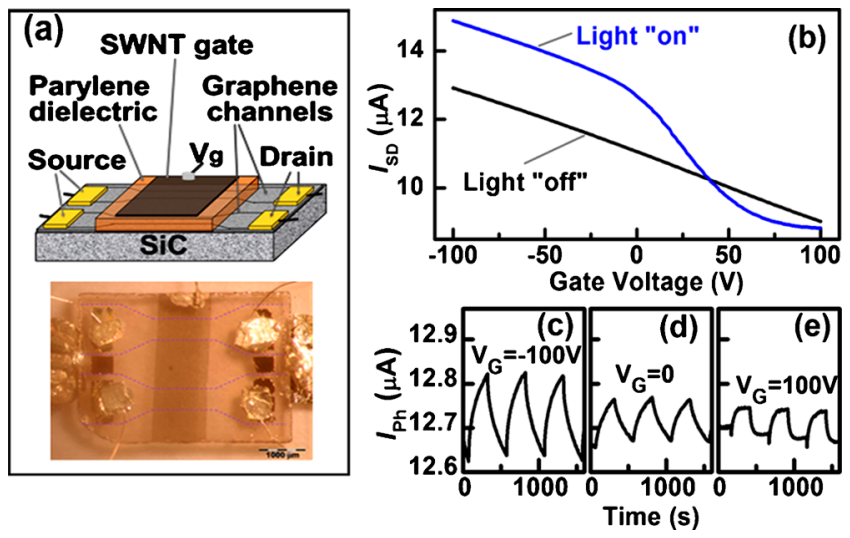

FIG. 3. (Color online) (a) Schematic (top) and microscope image of the SiC wafer with two FETs based on pristine and EO-EG channels with common SWNT thin film gate electrode. Dog bone shape of the channels is indicated by dashed line. (b) Modification of transfer characteristics of EO-EG channel FET under blue LED illumination $(\lambda=470 \mathrm{~nm})$ of incident power of $20 \mu \mathrm{W} .[(\mathrm{c})-(\mathrm{e})]$ Photoresponse of EO-EG channel to square-wave pulses of blue LED irradiation of incident power of $0.2 \mu \mathrm{W}$ at gate voltages (c) -100 $\mathrm{V}$, (d) $0 \mathrm{~V}$, and (e) $+100 \mathrm{~V}$.

seconds which enhances the photoconductivity-similar to the classical description of the effect of traps in semiconductors. ${ }^{16}$ The appearance of the surface states correlates with the enhancement of the Raman D band [Fig. 1(b)] which is due to the increased concentration of $s p^{3}$ carbon centers in the conjugated $s p^{2}$ network. In nonfunctionalized EG, the ambient environment can also induce surface states and enhance the photoconductivity when the number of layer is reduced to a single layer. ${ }^{17}$

With increasing incident power $P$, we observed a sublinear increase in photocurrent $I_{\mathrm{Ph}}$ [Fig. 2(b)], which can be expressed in the form: $I_{\mathrm{Ph}} \infty P^{\gamma}$, where $\gamma \sim 0.4$ [Fig. 2(b), inset]. Such dependence is typically observed when the traps levels are distributed over a wide energy range: an increase in the irradiation intensity forces the quasi-Fermi level to shift thus converting a number of traps into recombination centers. ${ }^{16}$ This leads to a decrease in the life time of the photoexcitations resulting in a sublinear dependence of the photocurrent on light intensity [Fig. 2(b)] and faster photoresponse at higher power level [Fig. 2(a)]. Similar transient photoconductivity phenomena have been observed in amorphous and microcrystalline silicon, ${ }^{18}$ amorphous semiconductors such as In-Ga-Zn-O (Ref. 19), and thin $\mathrm{ZnO}$ films and nanowires. ${ }^{20}$

For the fabrication of a field-effect transistor (FET) devices, the EO-EG sample was annealed in vacuum at $100{ }^{\circ} \mathrm{C}$ followed by deposition of a $1 \mu \mathrm{m}$ thick gate dielectric layer of parylene $\mathrm{N}$ by sublimation in vacuum. We did not observe any decrease in the photoresponse after the parylene coating, suggesting that the photoresponse does not originate from oxygen photodesorption. The FET fabrication was completed by placing a semitransparent conducting SWNT film on top of the parylene dielectric layer to serve as a gate electrode as shown in Fig. 3(a). ${ }^{15}$ Indium pads were used to connect the source and drain of the EG channels to the outside circuit and silver paste was used for contacting the SWNT gate electrode.

Figure 3(b) compares the transfer characteristics of the EO-EG FET in the dark and under blue LED illumination $(\lambda=470 \mathrm{~nm})$ with $20 \mu \mathrm{W}$ of incident power. In the absence 
of illumination the EO-EG channel shows strong p-type behavior as a result of the electro-oxidatively induced doping with neutrality point shifted to positive potentials which are beyond the maximum applied gate voltage of $V_{\mathrm{G}}=110 \mathrm{~V}$. Under illumination the on-off ratio increases from 1.4 to 1.7 and the minimum corresponding to the Dirac point becomes visible at $+100 \mathrm{~V}$. The carrier drift mobility $\mu$ was calculated from the source-drain current $I_{\mathrm{SD}}$ versus gate voltage slope, $d I_{\mathrm{SD}} / d V_{\mathrm{G}}, \quad \mu=\left[\left(L \cdot t_{\mathrm{PAR}}\right) /\right.$ $\left.\left(\varepsilon_{\mathrm{PAR}} \cdot \varepsilon_{0} \cdot V_{\mathrm{SD}} \cdot W\right)\right] \cdot d I_{\mathrm{SD}} / d V_{\mathrm{G}}$, where the channel length $L$ $=1.3 \mathrm{~mm}$, width $W=0.35 \mathrm{~mm}$; thickness $t_{\mathrm{PAR}}=1 \mu \mathrm{m}$, dielectric constants of vacuum $\varepsilon_{0}$ and parylene $\varepsilon_{\mathrm{PAR}}=2.65$ and, and the source-drain voltage $V_{\mathrm{SD}}=0.1 \mathrm{~V}$. In the dark, we obtained a mobility of $\mu=390 \mathrm{~cm}^{2} / \mathrm{V} \mathrm{s} .{ }^{15}$ Under illumination the slope of the transfer characteristic increases in the gate voltage range from 10 to $50 \mathrm{~V}$, corresponding to a factor of 3 increase in the drift mobility of the holes to $\mu$ $=1100 \mathrm{~cm}^{2} / \mathrm{V} \mathrm{s}$.

We also observed the reciprocal effect as shown in Figs. 3(c)-3(e): the amplitude of the photoresponse of the EO-EG FET channel depends on the FET gate voltage. A negative gate voltage of $V_{\mathrm{G}}=-100 \mathrm{~V}$ enhances the photoresponse by a factor of $\sim 2$ in comparison with $V_{\mathrm{G}}=0$ while a positive gate voltage $V_{\mathrm{G}}=100 \mathrm{~V}$ decreases the photoresponse by a factor of $\sim 2$. This behavior is consistent with the model presented above: the gate potential shifts the position of the Fermi level with respect to the energy levels of the traps, which converts a number of traps into recombination centers, thus changing the recombination dynamics. For positive gate voltages it decreases the photocarriers lifetime, which also leads to a decreased photoresponse [Fig. 3(e)] while a negative gate voltage prolongs the life time and increases the photoresponse [Fig. 3(c)].

Photoconductive properties are usually associated with semiconducting materials with a well-defined energy gap. Our finding of enhanced photosensitivity in semimetallic EG can be ascribed to release of photocarriers from bound states (deep traps) formed at the EG interface to EO-EG and a significantly prolonged life time of photoexcitations due to the effect of deep traps on the recombination dynamics. In contrast to the high speed photoresponse of intrinsic exfoliated graphene, ${ }^{4,5}$ EO-EG shows much higher sensitivity but a slow response time, thus suggesting a different scope of applications. Potential applications include UV detection for astronomy, biological, and environmental research, and a missile plume detection, which requires blindness in the visible spectral range. ${ }^{21,22}$ The enhanced photosensitivity and high selectivity in the UV spectral range make EO-EG an interesting alternative to the less spectrally selective $\mathrm{Si}$ for UV detection applications and to the wide band semiconductors UV photodetectors such as $\mathrm{GaN}, \mathrm{AlGaN}$, diamond, $\mathrm{SiC}$, $\mathrm{ZnO}, \mathrm{ZnMgO}, \mathrm{ZnSe}{ }^{20-22}$ although further optimization of the chemistry is required to shorten the photoresponse time while preserving high sensitivity.

In conclusion, we demonstrated that electro-oxidation of EG significantly enhances its photosensitivity, most probably due to the introduction of interface states. Different chemistries can induce appropriate modifications in the graphene electronic structure so as to convert semimetallic graphene into a graphene semiconductor with a true gap at the Fermi level and a strong, fast photoresponse in the infrared spectral ranges.

This study was supported by DOD/DMEA under Contract No. H94003-10-2-1003 and NSF-MRSEC through Contract No. DMR-0820382.

${ }^{1}$ C. Berger, Z. Song, T. Li, X. Li, A. Y. Ogbazghi, R. Feng, Z. Dai, A. N. Marchenkov, E. H. Conrad, P. N. First, and W. A. de Heer, J. Phys. Chem. B 108, 19912 (2004).

${ }^{2}$ A. K. Geim and K. S. Novoselov, Nature Mater. 6, 183 (2007).

${ }^{3}$ C. Berger, Z. M. Song, X. B. Li, X. S. Wu, N. Brown, C. Naud, D. Mayou, T. B. Li, J. Hass, A. N. Marchenkov, E. H. Conrad, P. N. First, and W. A de Heer, Science 312, 1191 (2006).

${ }^{4}$ F. Xia, T. Mueller, Y. Lin, A. Valdes-Garcia, and Ph. Avouris, Nat. Nanotechnol. 4, 839 (2009).

${ }^{5}$ T. Mueller, F. Xia, and Ph. Avouris, Nat. Photonics 4, 297 (2010).

${ }^{6}$ E. J. H. Lee, K. Balasubramanian, R. T. Weitz, M. Burghard, and K. Kern, Nat. Nanotechnol. 3, 486 (2008).

${ }^{7}$ J. Park, Y. H. Ahn, and C. Ruiz-Vargas, Nano Lett. 9, 1742 (2009).

${ }^{8}$ X. Lv, Y. Huang, Z. Liu, J. Tian, Y. Wang, Y. Ma, J. Liang, S. Fu, X. Wan, and Y. Chen, Small 5, 1682 (2009).

${ }^{9}$ S. Ghosh, B. K. Sarker, A. Chunder, L. Zhai, and S. I. Khondaker, Appl. Phys. Lett. 96, 163109 (2010).

${ }^{10}$ Y. Lin, K. Zhang, W. Chen, Y. Liu, Z. Geng, J. Zeng, N. Pan, L. Yan, X. Wang, and J. G. Hou, ACS Nano 4, 3033 (2010).

${ }^{11}$ X. Geng, L. Niu, Z. Xing, R. Song, G. Liu, M. Sun, G. Cheng, H. Zhong, Z. Liu, Z. Zhang, L. Sun, H. Xu, L. Lu, and L. Liu, Adv. Mater. 22, 638 (2010)

${ }^{12}$ S. Niyogi, M. A. Hamon, H. Hu, B. Zhao, P. Bhowmik, R. Sen, M. E. Itkis, and R. C. Haddon, Acc. Chem. Res. 35, 1105 (2002).

${ }^{13}$ E. Bekyarova, M. E. Itkis, P. Ramesh, C. Berger, M. Sprinkle, W. A. de Heer, and R. C. Haddon, J. Am. Chem. Soc. 131, 1336 (2009).

${ }^{14}$ S. Niyogi, E. Bekyarova, M. E. Itkis, H. Zhang, K. Shepperd, J. Hick, M. Sprinkle, C. Berger, C. N. Lau, W. A. de Heer, E. H. Conrad, and R. C. Haddon, Nano Lett. 10, 4061 (2010).

${ }^{15}$ P. Ramesh, M. E. Itkis, E. Bekyarova, F. Wang, S. Niyogi, X. Chi, C. Berger, W. A. de Heer, and R. C. Haddon, J. Am. Chem. Soc. 132, 14429 (2010).

${ }^{16}$ N. V. Joshi, Photoconductivity: Art, Science, and Technology (Marcel Dekker, New York, 1990).

${ }^{17}$ X. Wu, Y. Hu, M. Ruan, N. Madiomanana, J. Hankinson, M. Sprinkle, C. Berger, and W. A. de Heer, Appl. Phys. Lett. 95, 223108 (2009).

${ }^{18}$ R. Brüggemann, J. Mater. Sci.: Mater. Electron. 14, 629 (2003).

${ }^{19}$ D. H. Lee, K. Kawamura, K. Nomura, T. Kamiya, and H. Hosono, Electrochem. Solid-State Lett. 13, H324 (2010).

${ }^{20}$ H. G. Zheng, Q. Sh. Li, J. P. Zhao, D. Chen, B. Zhao, Y. J. Yang, and L. Ch. Zhang, Appl. Surf. Sci. 253, 2264 (2006).

${ }^{21}$ E. Monroy, F. Omnes, and F. Calle, Semicond. Sci. Technol. 18, R33 (2003).

${ }^{22}$ K. Liu, M. Sakurai, and M. Aono, Sensors 10, 8604 (2010). 